\title{
氧基亚胺与亚甲基二氢苯并呋喃二酮类化合物的[3+2]环加成反应: 螺吡唑类化合物的合成
}

\author{
史望宇徐嘉擎毛比明贾皓黄家兴* 郭红超*
}

(中国农业大学理学院化学系 北京 100193)

\begin{abstract}
摘要 采用具有环外双键的二氢苯并呋喃二酮类化合物和氰基亚胺的 $[3+2]$ 环加成反应，在温和条件下，以高产率 (78\% 94\%)和高非对映选择性( $>20: 1 d r)$ 构建了一系列具有螺环结构的吡唑类化合物.
\end{abstract}

关键词 环加成; 氰基亚胺; 螺环化合物; 吡唑

\section{[3+2] Cycloaddition of Methylenedihydrobenzofurandiones and Nitrilimines: Synthesis of Sipropyrazole Compounds}

\author{
Shi, Wangyu Xu, Jiaqing Mao, Biming Jia, Hao Huang, Jiaxing* Guo, Hongchao* \\ (Department of Chemistry, College of Science, China Agricultural University, Beijing 100193)
}

\begin{abstract}
The [3+2] cycloaddition of methylenedihydrobenzofurandiones and nitrilimines worked efficiently in $\mathrm{CHCl}_{3}$ at room temperature in the presence of $\mathrm{Et}_{3} \mathrm{~N}$, producing biologically interesting spirocyclic pyrazole derivatives in high yield (78\% 94\%) with excellent diastereoselectivity.
\end{abstract}

Keywords cycloaddition; nitrilimine; spirocyclic compound; pyrazole

吡唑类化合物具有多种生物活性, 例如除草、杀虫 和杀菌、抗癌、抗菌、抗氧化和 IRK4 抑制等作用 ${ }^{[1]}$, 含 有吡唑及其衍生结构的化合物已被广泛应用于农药和 医药开发中. 具有螺环结构的吡唑类化合物是一类重要 的吡唑衍生物, 含有这种结构单元的化合物已被证实具 有抗癌 ${ }^{[2]}$ 、酶抑制 ${ }^{[3]}$ 、除草 ${ }^{[4]}$ 和抗结核分岐杆菌 ${ }^{[3]}$ 等作用, 因此, 它们的合成研究受到了广泛关注. 1960 年, Huisgen 课题组 ${ }^{[6]}$ 通过氰基亚胺和偶氮次甲基亚胺的 [3+2] 热环化反应构建了一系列的螺环吡唑类化合物. 1993 年, Belletire 课题组 ${ }^{[7]}$ 报道了一种通过自由基机理合成 螺环吡唑类化合物的方法. 2014 年, 施敏课题组 ${ }^{[8]}$ 通过 钯催化反应, 合成了一种氮杂环状 1,3-偶极子, 并用这 种偶极子合成了一系列具有螺环结构的吡唑类衍生物, 为实现螺环吡唑类化合物的合成提供了新的途径.

环加成反应在杂环化合物合成中扮演着重要角色. 截至目前, 已有多种 1,3-偶极子被用于环加成反应中, 例如硝酮、偶氮次甲基亚胺、亚甲胺叶立德、羰基叶立
德、氰基叶立德、氰基亚胺、氰基氧化物、重氮化合物 和叠氮化合物等 ${ }^{[9]}$. 其中, 氧基亚胺作为一种重要的 1,3-偶极子，经常被用于含氮杂环化合物的合成 ${ }^{[10,11]}$. 在碱的存在下, $N$-芳基芳羰基亚朋酰氯脱去一分子氯化 氢, 形成氰基亚胺中间体(Scheme 1), 再与亲偶极体如 亚胺、烯烃、炔烃和烯胺等发生环加成反应, 得到杂环 化合物. 1962 年, Huisgen 课题组 ${ }^{[12]}$ 报道了氧基亚胺与亚 胺的 $[3+2]$ 环加成反应, 合成三氮唑类化合物. 1979 年, Tsuge 课题组 ${ }^{[13]}$ 报道了氰基亚胺与氮杂环丙烷的 $[3+3]$ 环加成反应，实现 1,2,4-三嗪类衍生物的合成. 同年, Gandolfi 课题组 ${ }^{[14]}$ 报道了氭基亚胺与环庚三烯亚胺 $[8+3]$ 环化反应, 完成了环庚三烯并 1,2,4-三嗪类化合 物的合成. 2018 年, 本课题组与王治永课题组 ${ }^{[15]}$ 相继报 道了氧基亚胺与氮杂邻亚甲基苯醌的 $[4+3]$ 环化反应, 实现 $[1,2,4]$ 三氮杂草衍生物的合成. 最近, 本组 ${ }^{[16]}$ 报道 了氧基亚胺与香豆灵酸甲酯或吡喃酮的 $[3+2]$ 环加成反 应, 以较高至优秀的产率和优秀的非对映选择性合成二

* Corresponding authors. E-mail: hchguo@cau.edu.cn; 05084@cau.edu.cn Received July 22, 2019; revised October 18, 2019; published online November 1, 2019 Project supported by the National Natural Science Foundation of China (No. 21572264). 国家自然科学基金(No. 21572264)资助项目. 
氢吡喃并吡唑酮衍生物. 基于我们之前的工作 ${ }^{[15 ~ 17]}$, 本 工作采用氰基亚胺与具有环外双键的二氢苯并呋喃二 酩进行环加成反应, 高产率高立体选择性地合成了螺吡 唑类化合物(Scheme 1).

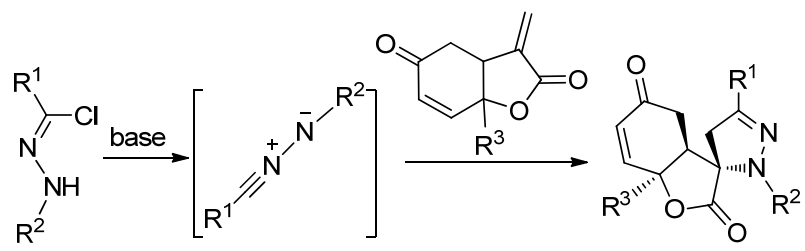

图式 1 氰基亚胺与具有环外双键的二氢苯并呋喃二酮的环 加成反应

Scheme 1 Cycloaddition reactions between methylenedihydrobenzofurandione and nitrilimines

\section{1 结果与讨论}

最初使用三乙烯二胺(DABCO)为碱, 探索了二氢 苯并呋喃二酮类化合物 $\mathbf{1 a}$ 和 $N$-芳基芳羰基亚肼酰氯 $\mathbf{2 a}$ 的 $[3+2]$ 环加成反应, 以二氯甲烷(DCM) 为溶剂, 在室 温下反应 $24 \mathrm{~h}$, 以 $72 \%$ 的产率得到所期望的产物 $3 \mathbf{a a}$ (表 1, Entry 1). 接下来, 分别篎选了 1,8-二氮杂二环十一 碳-7-烯( $\mathrm{DBU}) 、$ 三乙胺 $\left(\mathrm{Et}_{3} \mathrm{~N}\right)$ 和二异丙基乙基胺 $\left(i-\mathrm{Pr}_{2}-\right.$ NEt) 三种有机碱(Entries 2 4), 分别以 $69 \% 、 86 \%$ 和 75\% 的产率得到目标产物. 作为对比, 使用碳酸铯和

表 1 环加成反应条件篎选 ${ }^{a}$

Table 1 Screening of the reaction conditions

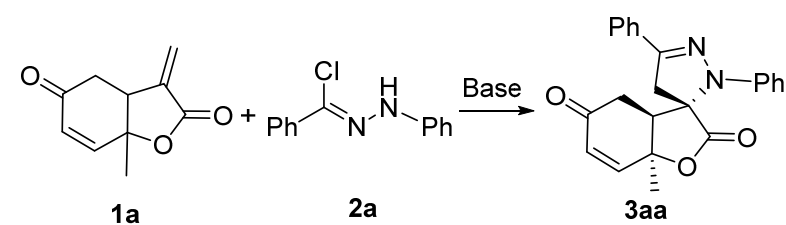

\begin{tabular}{cllc}
\hline Entry & \multicolumn{1}{c}{ Base } & Solvent & Yield $^{b} \%$ \\
\hline 1 & $\mathrm{DABCO}$ & $\mathrm{DCM}$ & 72 \\
2 & $\mathrm{DBU}$ & $\mathrm{DCM}$ & 69 \\
3 & $\mathrm{Et}_{3} \mathrm{~N}$ & $\mathrm{DCM}$ & 86 \\
4 & $i-\mathrm{Pr}_{2} \mathrm{NEt}$ & $\mathrm{DCM}$ & 75 \\
5 & $\mathrm{~K}_{2} \mathrm{CO}_{3}$ & $\mathrm{DCM}$ & 60 \\
6 & $\mathrm{Cs}_{2} \mathrm{CO}_{3}$ & $\mathrm{DCM}$ & 78 \\
7 & $\mathrm{Et}_{3} \mathrm{~N}$ & $\mathrm{DCE}$ & 81 \\
8 & $\mathrm{Et}_{3} \mathrm{~N}$ & $\mathrm{Toluene}$ & 67 \\
9 & $\mathrm{Et}_{3} \mathrm{~N}$ & $\mathrm{CH} C \mathrm{CN}$ & 75 \\
10 & $\mathrm{Et}_{3} \mathrm{~N}$ & $\mathrm{THF}$ & 58 \\
11 & $\mathrm{Et}_{3} \mathrm{~N}$ & $\mathrm{MeOH}$ & 78 \\
12 & $\mathrm{Et}_{3} \mathrm{~N}$ & $\mathrm{CHCl}_{3}$ & 89 \\
$13^{c}$ & $\mathrm{Et}_{3} \mathrm{~N}$ & $\mathrm{CHCl}_{3}$ & 93 \\
\hline
\end{tabular}

${ }^{a}$ Reaction conditions: 1a $(0.1 \mathrm{mmol}) 、 2 a(0.1 \mathrm{mmol})$, base $(0.12 \mathrm{mmol})$, room temperature, $24 \mathrm{~h} ;{ }^{b}$ Isolated yields, $d r(\mathbf{3})>20: 1 ;{ }^{c} \mathbf{2 a}(0.12 \mathrm{mmol}), 12 \mathrm{~h}$.
碳酸钾这两种无机碱分别得到了 $60 \%$ 和 $78 \%$ 的产率 (Entries 5,6). 从上述结果可以看出, 所有碱均能以较高 产率得到目标产物. 相比于无机碱, 有机碱的效果更好, 产率相对较高. 在所有测试的碱中, 采用三乙胺可以得 到最高的产率，因此，选定三乙胺作为碱，对溶剂和两 种反应物的比例进行了耖选(Entries 7 13). 根据篮选 结果，以三氯甲烷作为溶剂、三乙胺作为碱，使用 1.2 equiv. 的 $N$-芳基芳羰基亚肼酰氯(2a) 时得到了最高产率 (93\%). 通过 X 射线单晶衍射确定了产物 3aa (CCDC: 1937319)的相对构型.

在确立了最优条件之后，对苯环上不同取代基的 $N$-芳基芳羰基亚肼酰氯进行了探索，测试反应底物的适 用性，结果如表 2 所示. 芳香基 $\mathrm{R}^{1}$ 上为卤素取代基时， $[3+2]$ 环加成反应以良好到优秀产率得到对应产物 3ab 3ah (Entries 2 7, 产率 83\% 94\%). $\mathrm{R}^{1}$ 上的取代 基为给电子基团时，反应仍可高效进行(Entry 8，产率 $91 \%$ ). 当 $\mathrm{R}^{1}$ 为 2 -萗基时，以 $78 \%$ 的产率得到螺环产物 (Entry 9). 芳香基 $\mathrm{R}^{2}$ 为卤素或甲基取代基时，氰基亚胺 底物均兼容该反应，以良好到优秀产率及优秀的非对映

表 $2 \mathrm{~N}$-芳基芳羰基亚肼酰氯 2 的范围 ${ }^{a}$

Table 2 Substrate scope of the arylcarbohydrazonoyl chlorides 2

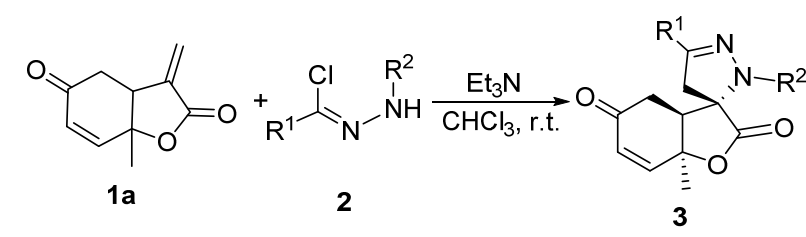

\begin{tabular}{|c|c|c|c|c|c|}
\hline Entry & $\mathrm{R}^{1}$ & $\mathrm{R}^{2}$ & $t / \mathrm{h}$ & 3 & Yield $^{b} / \%$ \\
\hline 1 & $\mathrm{Ph}$ & $\mathrm{Ph}$ & 12 & $3 \mathbf{a a}$ & 93 \\
\hline 2 & $2-\mathrm{FC}_{6} \mathrm{H}_{4}$ & $\mathrm{Ph}$ & 12 & $3 a b$ & 93 \\
\hline $3^{c}$ & $3-\mathrm{FC}_{6} \mathrm{H}_{4}$ & $\mathrm{Ph}$ & 12 & $3 a c$ & 83 \\
\hline 4 & $4-\mathrm{FC}_{6} \mathrm{H}_{4}$ & $\mathrm{Ph}$ & 14 & 3ad & 84 \\
\hline 8 & $3-\mathrm{ClC}_{6} \mathrm{H}_{4}$ & $\mathrm{Ph}$ & 14 & $3 a e$ & 94 \\
\hline 5 & $2-\mathrm{BrC}_{6} \mathrm{H}_{4}$ & $\mathrm{Ph}$ & 14 & 3af & 93 \\
\hline 6 & $3-\mathrm{BrC}_{6} \mathrm{H}_{4}$ & $\mathrm{Ph}$ & 14 & $3 a g$ & 88 \\
\hline 7 & $4-\mathrm{BrC}_{6} \mathrm{H}_{4}$ & $\mathrm{Ph}$ & 24 & 3ah & 91 \\
\hline 8 & $2-\mathrm{MeC}_{6} \mathrm{H}_{4}$ & $\mathrm{Ph}$ & 24 & 3ai & 91 \\
\hline 9 & 2-Naphthyl & $\mathrm{Ph}$ & 12 & 3aj & 78 \\
\hline 10 & $\mathrm{Ph}$ & $2-\mathrm{FC}_{6} \mathrm{H}_{4}$ & 24 & 3ak & 85 \\
\hline 11 & $\mathrm{Ph}$ & $2-\mathrm{ClC}_{6} \mathrm{H}_{4}$ & 24 & 3al & 80 \\
\hline 12 & $\mathrm{Ph}$ & $3-\mathrm{ClC}_{6} \mathrm{H}_{4}$ & 24 & 3am & 90 \\
\hline 13 & $\mathrm{Ph}$ & $3-\mathrm{BrC}_{6} \mathrm{H}_{4}$ & 24 & 3an & 94 \\
\hline 14 & $\mathrm{Ph}$ & $2-\mathrm{MeC}_{6} \mathrm{H}_{4}$ & 48 & 3 ao & 85 \\
\hline 15 & $\mathrm{Ph}$ & 4- $\mathrm{MeC}_{6} \mathrm{H}_{4}$ & 14 & 3 ap & 89 \\
\hline
\end{tabular}

${ }^{a}$ Reaction conditions: 1a $(0.1 \mathrm{mmol}), 2(0.12 \mathrm{mmol}), \mathrm{Et}_{3} \mathrm{~N}(0.12 \mathrm{mmol})$, room temperature. ${ }^{b}$ Isolated yields. ${ }^{c} d r=16: 1$. 
选择性得到目标产物(Entries $10 \sim 15$, 产率 $80 \% \sim 94 \%$, $d r>20: 1)$. 除产物 $3 \mathbf{a c}$ 外, 所有螺环产物的非对映异 构体的比例均大于 $20: 1$.

在完成了对 $N$-芳基芳羰基亚肼酰氯底物的范围调 查后, 又对二氢苯并呋喃二酮底物进行了探索 (Scheme 2). 在 $\mathrm{R}$ 位引入乙基或苯基时，分别以 $78 \%$ 和 $85 \%$ 的产 率以及大于 $20: 1 d r$ 值得到产物 $3 \mathbf{b a}$ 和 $3 \mathbf{c a}$. 这表明在 $\mathrm{R}$ 位引入新的官能团并不影响反应的产率和立体选择 性，证明了该反应具有较好的底物兼容性.

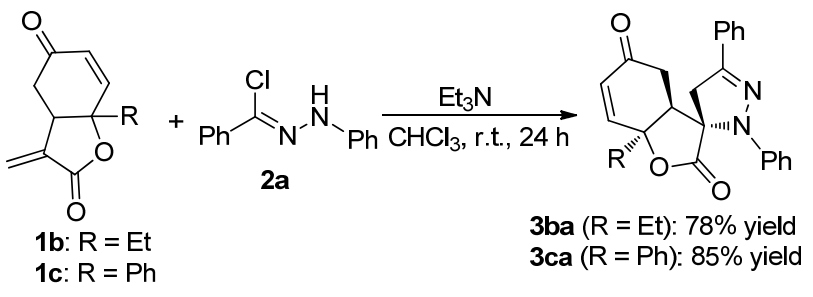

图式 2 亚甲基二氢苯并呋喃二酮 1 的范围 Scheme 2 Substrate scope of methylenedihydrobenzofurandiones 1

\section{2 结论}

通过二氢苯并呋喃二酮化合物和氰基亚胺的 $[3+2]$ 环加成反应, 在温和条件下高产率高非对映选择性地构 建了一系列具有潜在生物活性的螺吡唑类化合物. 这些 螺环化合物的生物活性目前正在测试中.

\section{3 实验部分}

\section{1 仪器与试剂}

${ }^{1} \mathrm{H}$ NMR 和 ${ }^{13} \mathrm{C}$ NMR 数据通过 Bruker 300 核磁仪 测定, 内标为 TMS. HRMS 数据通过 ESI-MS 技术测得. 所有反应用磁力搅拌器搅拌, 在烘干的玻璃仪器中氩气 保护下进行.

所有试剂均通过商业购买, 无需进一步纯化. 所有 溶剂经无水无氧处理. 有机溶剂通过减压旋转蒸发及油 洜除去. 反应通过薄层色谱(TLC)检测, 通过硅胶柱色 谱分离纯化, 硅胶使用青岛海洋硅胶(中性, 200 300 目).

\section{2 实验方法}

\section{2 .1 底物合成方法}

二氢苯并呋喃二酮类化合物 $1 \mathbf{a} \sim 1 \mathrm{c}$ 合成方法参考 文献[18], $N$-芳基芳羰基亚肼酰氯的合成方法参考文献 [19].

\subsection{2 螺环吡唑化合物 3 的合成}

氩气保护下向反应管中依次加入 $0.1 \mathrm{mmol}$ 的二氢 苯并呋喃二酮(1)、0.12 mmol 的 $N$-芳基芳羰基亚肼酰氯
(2)、 $0.12 \mathrm{mmol}$ 的 $\mathrm{Et}_{3} \mathrm{~N}$ 和 $1 \mathrm{~mL}$ 的三氯甲烷，在 $25{ }^{\circ} \mathrm{C}$ 水浴下反应, TLC 跟踪反应, 待反应物 1 完全消耗，直接 经柱层析(石油醚/乙酸乙酯, $V: V=4: 1$ )纯化得化合物 3.

7a-甲基-2',5'-二苯基-2',3a,4',7a-四氢-2 $H$-螺[苯并呋 喃-3,3'-吡唑]-2,5(4H)-二酮(3aa): 淡黄色固体, $34.6 \mathrm{mg}$, 产率 93\%. m.p. $125 \sim 127{ }^{\circ} \mathrm{C}$; ${ }^{1} \mathrm{H}$ NMR $(300 \mathrm{MHz}$, $\left.\mathrm{CDCl}_{3}\right) \delta: 7.66 \sim 7.56(\mathrm{~m}, 2 \mathrm{H}), 7.44 \sim 7.30(\mathrm{~m}, 5 \mathrm{H}), 7.16$ (dd, $J=8.7,1.0 \mathrm{~Hz}, 2 \mathrm{H}), 7.01(\mathrm{t}, J=7.3 \mathrm{~Hz}, 1 \mathrm{H}), 6.83$ (dd, $J=10.4,1.6 \mathrm{~Hz}, 1 \mathrm{H}), 6.19$ (d, $J=10.4 \mathrm{~Hz}, 1 \mathrm{H}), 3.60 \sim$ $3.31(\mathrm{~m}, 3 \mathrm{H}), 2.65$ (d, J=4.5 Hz, 2H), $1.74(\mathrm{~s}, 3 \mathrm{H}) ;{ }^{13} \mathrm{C}$ NMR $\left(75 \mathrm{MHz}, \mathrm{CDCl}_{3}\right) \delta$ : 194.1, 174.0, 147.3, 145.7, $141.5,130.7,129.11,129.07,129.0,128.4,125.6,121.5$, 116.5, 78.6, 73.2, 42.4, 40.9, 33.2, 25.6; HRMS (ESI) calcd for $\mathrm{C}_{23} \mathrm{H}_{21} \mathrm{~N}_{2} \mathrm{O}_{3}[\mathrm{M}+\mathrm{H}]^{+}$373.1547, found 373.1551 .

5'-(2-氟苯基)-7a-甲基-2'-苯基-2',3a,4',7a-四氢- $2 H$ 螺 [苯并呋喃-3,3'-吡唑]-2,5(4H)-二酮(3ab): 淡黄色固 体, $36.5 \mathrm{mg}$, 产率 93\%. m.p. 188 $190{ }^{\circ} \mathrm{C} ;{ }^{1} \mathrm{H}$ NMR $(300$ $\left.\mathrm{MHz}, \mathrm{CDCl}_{3}\right) \delta: 7.92(\mathrm{td}, J=7.8,1.8 \mathrm{~Hz}, 1 \mathrm{H}), 7.32$ (ddd, $J=7.4,6.9,1.9 \mathrm{~Hz}, 3 \mathrm{H}), 7.22 \sim 7.11(\mathrm{~m}, 3 \mathrm{H}), 7.11 \sim 6.96$ (m, 2H), $6.82(\mathrm{dd}, J=10.4,1.7 \mathrm{~Hz}, 1 \mathrm{H}), 6.17$ (d, $J=10.4$ $\mathrm{Hz}, 1 \mathrm{H}), 3.66 \sim 3.48(\mathrm{~m}, 3 \mathrm{H}), 2.65(\mathrm{~d}, J=4.5 \mathrm{~Hz}, 2 \mathrm{H})$, $1.73(\mathrm{~s}, 3 \mathrm{H}) ;{ }^{13} \mathrm{C} \mathrm{NMR}\left(75 \mathrm{MHz}, \mathrm{CDCl}_{3}\right) \delta: 193.7,174.0$, 160.0 (d, $J=251.2 \mathrm{~Hz}), 147.2,142.1$ (d, $J=2.3 \mathrm{~Hz}), 141.2$, $130.6(\mathrm{~d}, J=8.5 \mathrm{~Hz}), 129.14,129.09,128.1$ (d, $J=3.2 \mathrm{~Hz})$, $124.1(\mathrm{~d}, J=3.0 \mathrm{~Hz}), 121.7,118.7$ (d, $J=11.1 \mathrm{~Hz}), 116.5$, $116.1(\mathrm{~d}, J=22.2 \mathrm{~Hz}), 78.7,73.2,42.9$ (d, $J=9.0 \mathrm{~Hz})$, 42.4, 33.0, 25.7; HRMS (ESI) calcd for $\mathrm{C}_{23} \mathrm{H}_{20} \mathrm{FN}_{2} \mathrm{O}_{3}$ $[\mathrm{M}+\mathrm{H}]^{+}$391.1452, found 391.1449.

5'-(3-氟苯基)-7a-甲基-2'-苯基-2',3a,4',7a-四氢- $2 H$ 螺 [苯并呋喃-3,3'-吡唑]-2,5(4H)-二酮(3ac): 白色固体, $32.4 \mathrm{mg}$, 产率 $83 \%$. m.p. $185 \sim 187{ }^{\circ} \mathrm{C}$; ${ }^{1} \mathrm{H}$ NMR $(300$ $\mathrm{MHz} \mathrm{CDCl}_{3}$ ) $\delta: 7.34$ (qdd, $\left.J=11.8,5.8,4.4 \mathrm{~Hz}, 5 \mathrm{H}\right), 7.15$ (dd, $J=8.7,1.0 \mathrm{~Hz}, 2 \mathrm{H}), 7.05$ (ddd, $J=14.7,6.3,4.3 \mathrm{~Hz}$, 2H), 6.83 (dd, $J=10.4,1.6 \mathrm{~Hz}, 1 \mathrm{H}), 6.19$ (d, $J=10.4 \mathrm{~Hz}$, $1 \mathrm{H}), 3.62 \sim 3.24(\mathrm{~m}, 3 \mathrm{H}), 2.76 \sim 2.53(\mathrm{~m}, 2 \mathrm{H}), 1.74(\mathrm{~s}$, $3 \mathrm{H}) ;{ }^{13} \mathrm{C} \mathrm{NMR}\left(75 \mathrm{MHz}, \mathrm{CDCl}_{3}\right) \delta: 194.1,173.8,162.6(\mathrm{~d}$, $J=246.3 \mathrm{~Hz}), 147.2,144.5$ (d, $J=3.0 \mathrm{~Hz}), 141.1,132.8$ (d, $J=8.3 \mathrm{~Hz}), 130.0$ (d, $J=8.3 \mathrm{~Hz}), 129.2,129.0,121.9$, $121.2(\mathrm{~d}, J=2.8 \mathrm{~Hz}), 116.6,116.0$ (d, $J=21.5 \mathrm{~Hz}), 112.2$ (d, $J=23.0 \mathrm{~Hz}$ ), 78.7, 73.4, 42.4, 40.7, 33.2, 30.6, 25.6; HRMS (ESI) calcd for $\mathrm{C}_{23} \mathrm{H}_{20} \mathrm{FN}_{2} \mathrm{O}_{3}[\mathrm{M}+\mathrm{H}]^{+}$391.1452, found 391.1458 .

5'-(4-氟苯基)-7a-甲基-2'-苯基-2',3a,4',7a-四氢- $2 H$ - 
螺 [苯并呋喃-3,3'-吡唑]-2,5(4H)-二酮(3ad): 淡黄色固 体, $32.6 \mathrm{mg}$, 产率 84\%. m.p. 191 193 ${ }^{\circ} \mathrm{C} ;{ }^{1} \mathrm{H}$ NMR $(300$ $\left.\mathrm{MHz}, \mathrm{CDCl}_{3}\right) \delta: 7.64 \sim 7.53(\mathrm{~m}, 2 \mathrm{H}), 7.33(\mathrm{dd}, J=8.6,7.4$ $\mathrm{Hz}, 2 \mathrm{H}), 7.19 \sim 6.96(\mathrm{~m}, 5 \mathrm{H}), 6.82(\mathrm{dd}, J=10.4,1.6 \mathrm{~Hz}$, $1 \mathrm{H}), 6.18(\mathrm{~d}, J=10.4 \mathrm{~Hz}, 1 \mathrm{H}), 3.53 \sim 3.30(\mathrm{~m}, 3 \mathrm{H}), 2.76 \sim$ $2.53(\mathrm{~m}, 2 \mathrm{H}), 1.73(\mathrm{~s}, 3 \mathrm{H}) ;{ }^{13} \mathrm{C} \mathrm{NMR}\left(75 \mathrm{MHz}, \mathrm{CDCl}_{3}\right) \delta$ : 194.2, 173.9, 163.1 (d, $J=250.1 \mathrm{~Hz}), 147.3,144.8,141.4$, 129.1, 129.0, 127.4 (d, $J=8.4 \mathrm{~Hz}), 127.0$ (d, $J=3.2 \mathrm{~Hz})$, 121.6, 116.5, 115.5 (d, $J=22.0 \mathrm{~Hz}$ ), 78.7, 73.4, 42.3, 41.0, 33.3, 25.6; HRMS (ESI) calcd for $\mathrm{C}_{23} \mathrm{H}_{20} \mathrm{FN}_{2} \mathrm{O}_{3}[\mathrm{M}+\mathrm{H}]^{+}$ 391.1452 , found 391.1451 .

5'-(3-氯苯基)-7a-甲基-2'-苯基-2',3a,4',7a-四氢- $2 H$ 螺[苯并呋喃-3,3'-吡唑]-2,5(4H)-二酮(3ae): 白色固体, $38.1 \mathrm{mg}$, 产率 94\%. m.p. 209 211 ${ }^{\circ} \mathrm{C} ;{ }^{1} \mathrm{H}$ NMR (300 $\left.\mathrm{MHz}, \mathrm{CDCl}_{3}\right) \delta: 7.64(\mathrm{~d}, J=2.0 \mathrm{~Hz}, 1 \mathrm{H}), 7.45 \sim 7.30(\mathrm{~m}$, $5 \mathrm{H}), 7.16(\mathrm{dd}, J=8.7,1.0 \mathrm{~Hz}, 2 \mathrm{H}), 7.03$ (t, $J=7.3 \mathrm{~Hz}$, $1 \mathrm{H}), 6.84(\mathrm{dd}, J=10.4,1.6 \mathrm{~Hz}, 1 \mathrm{H}), 6.20(\mathrm{~d}, J=10.4 \mathrm{~Hz}$, $1 \mathrm{H}), 3.72 \sim 3.58(\mathrm{~m}, 2 \mathrm{H}), 3.54 \sim 3.51(\mathrm{~m}, 1 \mathrm{H}), 2.78 \sim 2.53$ (m, 2H), $1.74(\mathrm{~s}, 3 \mathrm{H}) ;{ }^{13} \mathrm{C}$ NMR (151 MHz, $\left.\mathrm{CDCl}_{3}\right) \delta$ : 194.0, 173.6, 147.1, 144.1, 141.0, 134.4, 132.3, 129.5, $129.0,128.9,128.8,125.2,123.4,121.8,116.5,78.6,73.3$, 42.3, 40.5, 33.1, 30.5, 25.5; HRMS (ESI) calcd for $\mathrm{C}_{23} \mathrm{H}_{20} \mathrm{ClN}_{2} \mathrm{O}_{3}[\mathrm{M}+\mathrm{H}]^{+}$407.1157, found 407.1160.

$5^{\prime}$-(2-溴苯基)-7a-甲基-2'-苯基-2',3a, 4 ', $7 \mathrm{a}$-四氢- $2 \mathrm{H}$ 螺[苯并呋喃-3,3'-吡唑]-2,5(4H)-二酮(3af): 淡黄色固体, $42.0 \mathrm{mg}$, 产率 93\%. m.p. $129 \sim 131{ }^{\circ} \mathrm{C} ;{ }^{1} \mathrm{H}$ NMR $(300$ $\mathrm{MHz}, \mathrm{CDCl}_{3}$ ) $\delta: 7.59$ (ddd, $J=11.2,7.9,1.3 \mathrm{~Hz}, 2 \mathrm{H}$ ), 7.34 (dd, $J=11.1,4.7 \mathrm{~Hz}, 3 \mathrm{H}$ ), 7.19 (ddd, $J=14.0,9.9,4.8 \mathrm{~Hz}$, 3H), 7.01 (t, $J=7.3 \mathrm{~Hz}, 1 \mathrm{H}), 6.81$ (dd, $J=10.4,1.6 \mathrm{~Hz}$, $1 \mathrm{H}), 6.15(\mathrm{~d}, J=10.4 \mathrm{~Hz}, 1 \mathrm{H}), 3.74 \sim 3.57(\mathrm{~m}, 2 \mathrm{H}), 3.56 \sim$ $3.49(\mathrm{~m}, 1 \mathrm{H}), 2.83 \sim 2.61(\mathrm{~m}, 2 \mathrm{H}), 1.73(\mathrm{~s}, 3 \mathrm{H}) ;{ }^{13} \mathrm{C} \mathrm{NMR}$ $\left(75 \mathrm{MHz}, \mathrm{CDCl}_{3}\right) \delta: 193.6,173.9,147.2,145.4,141.2$, $133.8,131.9,130.3,130.0,129.2,129.1,127.2,121.8$, 120.9, 116.6, 78.6, 73.6, 43.4, 42.4, 33.1, 25.7; HRMS (ESI) calcd for $\mathrm{C}_{23} \mathrm{H}_{20} \mathrm{BrN}_{2} \mathrm{O}_{3} \quad[\mathrm{M}+\mathrm{H}]^{+}$453.0634, found 451.0586, 453.0631.

5'-(3-溴苯基)-7a-甲基-2'-苯基-2',3a, 4',7a-四氢- $2 \mathrm{H}$ 螺[苯并呋喃-3,3'-吡唑]-2,5(4H)-二酮(3ag): 淡黄色固体, $39.7 \mathrm{mg}$, 产率 $88 \%$. m.p. $216 \sim 218{ }^{\circ} \mathrm{C} ;{ }^{1} \mathrm{H}$ NMR $(300$ $\left.\mathrm{MHz}, \mathrm{CDCl}_{3}\right) \delta: 7.80(\mathrm{t}, J=1.7 \mathrm{~Hz}, 1 \mathrm{H}), 7.50 \sim 7.43(\mathrm{~m}$, 2H), $7.35(\mathrm{dd}, J=8.5,7.5 \mathrm{~Hz}, 2 \mathrm{H}), 7.23(\mathrm{~d}, J=7.9 \mathrm{~Hz}$, 1H), $7.16(\mathrm{dd}, J=8.7,0.9 \mathrm{~Hz}, 2 \mathrm{H}), 7.03$ (t, $J=7.3 \mathrm{~Hz}$, $1 \mathrm{H}), 6.84$ (dd, $J=10.4,1.6 \mathrm{~Hz}, 1 \mathrm{H}), 6.20$ (d, $J=10.4 \mathrm{~Hz}$, $1 \mathrm{H}), 3.61 \sim 3.30(\mathrm{~m}, 3 \mathrm{H}), 2.76 \sim 2.55(\mathrm{~m}, 2 \mathrm{H}), 1.75(\mathrm{~s}$, $3 \mathrm{H}) ;{ }^{13} \mathrm{C}$ NMR (101 MHz, $\left.\mathrm{CDCl}_{3}\right) \delta: 207.3,194.5,174.2$,
$147.7,144.5,141.4,133.1,132.3,130.3,129.6,128.7$, 124.4, 123.0, 122.3, 117.0, 79.2, 73.8, 42.8, 41.0, 33.6, 31.0, 26.0; HRMS (ESI) calcd for $\mathrm{C}_{23} \mathrm{H}_{20} \mathrm{BrN}_{2} \mathrm{O}_{3}[\mathrm{M}+\mathrm{H}]^{+}$ 453.0634, found 451.0614, 453.0637 .

5'-(4-溴苯基)-7a-甲基-2'-苯基-2',3a, 4',7a-四氢- $2 H$ 螺[苯并呋喃-3,3'-吡唑]-2,5(4H)-二酩(3ah): 白色固体, $41.1 \mathrm{mg}$, 产率 91\%. m.p. $199 \sim 201{ }^{\circ} \mathrm{C}$; ${ }^{1} \mathrm{H}$ NMR (300 $\left.\mathrm{MHz}, \mathrm{CDCl}_{3}\right) \delta: 7.55 \sim 7.41$ (m, 4H), 7.34 (tt, $J=4.1,2.1$ $\mathrm{Hz}, 2 \mathrm{H}), 7.18 \sim 7.09(\mathrm{~m}, 2 \mathrm{H}), 7.07 \sim 6.96(\mathrm{~m}, 1 \mathrm{H}), 6.83$ (dd, $J=10.4,1.7 \mathrm{~Hz}, 1 \mathrm{H}), 6.18(\mathrm{~d}, J=10.4 \mathrm{~Hz}, 1 \mathrm{H})$, $3.53 \sim 3.30(\mathrm{~m}, 3 \mathrm{H}), 2.77 \sim 2.51(\mathrm{~m}, 2 \mathrm{H}), 1.73(\mathrm{~s}, 3 \mathrm{H}) ;{ }^{13} \mathrm{C}$ NMR $\left(75 \mathrm{MHz}, \mathrm{CDCl}_{3}\right) \delta: 194.1,173.8,147.3,144.6$, $141.2,131.6,129.6,129.2,129.0,126.9,123.2,121.8$, 116.6, 78.7, 73.5, 42.4, 40.7, 33.3, 25.6; HRMS (ESI) calcd for $\mathrm{C}_{23} \mathrm{H}_{20} \mathrm{BrN}_{2} \mathrm{O}_{3}[\mathrm{M}+\mathrm{H}]^{+}$453.0634, found 451.0633, 453.0638.

7a-甲基-2'-苯基-5'-(邻甲基苯基)-2',3a,4',7a-四氢- $2 \mathrm{H}$ 螺[苯并呋喃-3,3'-吡唑]-2,5(4H)-二酮(3ai): 淡黄色固体, $35.3 \mathrm{mg}$, 产率 $91 \%$. m.p. $162 \sim 164{ }^{\circ} \mathrm{C} ;{ }^{1} \mathrm{H}$ NMR $(300$ $\left.\mathrm{MHz}, \mathrm{CDCl}_{3}\right) \delta: 7.57(\mathrm{dt}, J=4.5,2.6 \mathrm{~Hz}, 2 \mathrm{H}), 7.40 \sim 7.30$ (m, 3H), 7.28 (s, 1H), $7.25 \sim 7.12(\mathrm{~m}, 3 \mathrm{H}), 6.79$ (dd, $J=$ $10.4,1.7 \mathrm{~Hz}, 1 \mathrm{H}), 6.16$ (d, $J=10.4 \mathrm{~Hz}, 1 \mathrm{H}), 3.28$ (q, $J=$ $16.8 \mathrm{~Hz}, 2 \mathrm{H}), 3.01(\mathrm{~d}, J=6.8 \mathrm{~Hz}, 1 \mathrm{H}), 2.85 \sim 2.60(\mathrm{~m}$, $2 \mathrm{H}), 2.35(\mathrm{~s}, 3 \mathrm{H}), 1.58(\mathrm{~s}, 3 \mathrm{H}) ;{ }^{13} \mathrm{C}$ NMR $(75 \mathrm{MHz}$, $\left.\mathrm{CDCl}_{3}\right) \delta: 194.2,173.8,147.5,145.5,140.2,135.2,131.5$, $131.2,128.9,128.7,128.2,126.5,126.3,125.3,125.1$, 78.3, 74.9, 43.3, 40.1, 33.5, 25.8, 19.2; HRMS (ESI) calcd for $\mathrm{C}_{24} \mathrm{H}_{23} \mathrm{~N}_{2} \mathrm{O}_{3}[\mathrm{M}+\mathrm{H}]^{+}$387.1703, found 387.1669.

7a-甲基-5'-(芸-2-基)-2'-苯基-2',3a,4',7a-四氢- $2 \mathrm{H}$-螺 [苯并呋喃-3,3'-吡唑]-2,5(4H)-二酮(3aj): 白色固体, 33.1 mg, 产率 78\%. m.p. $187 \sim 189{ }^{\circ} \mathrm{C} ;{ }^{1} \mathrm{H}$ NMR $(300 \mathrm{MHz}$, $\left.\mathrm{CDCl}_{3}\right) \delta: 8.00(\mathrm{dd}, J=8.7,1.6 \mathrm{~Hz}, 1 \mathrm{H}), 7.90 \sim 7.78(\mathrm{~m}$, $3 \mathrm{H}), 7.72(\mathrm{~s}, 1 \mathrm{H}), 7.55 \sim 7.44(\mathrm{~m}, 2 \mathrm{H}), 7.36(\mathrm{t}, J=8.0 \mathrm{~Hz}$, 2H), $7.21(\mathrm{~d}, J=7.7 \mathrm{~Hz}, 2 \mathrm{H}), 7.02(\mathrm{t}, J=7.3 \mathrm{~Hz}, 1 \mathrm{H}), 6.85$ (dd, $J=10.4,1.5 \mathrm{~Hz}, 1 \mathrm{H}), 6.23(\mathrm{~d}, J=10.4 \mathrm{~Hz}, 1 \mathrm{H})$, $3.76 \sim 3.37$ (m, 3H), 2.67 (d, $J=4.8 \mathrm{~Hz}, 2 \mathrm{H}), 1.75$ (s, 3H); ${ }^{13} \mathrm{C}$ NMR $\left(75 \mathrm{MHz}, \mathrm{CDCl}_{3}\right) \delta: 194.2,174.0,147.3,145.8$, $141.4,133.4,132.8,129.2,129.1,128.3,128.2,128.0$, $127.5,126.5,126.3,125.1,123.0,121.6,116.5,78.7,73.3$, 42.4, 40.9, 33.2, 25.6; HRMS (ESI) calcd for $\mathrm{C}_{27} \mathrm{H}_{23} \mathrm{~N}_{2} \mathrm{O}_{3}$ $[\mathrm{M}+\mathrm{H}]^{+}$445.1523, found 445.1525.

2'-(2-氟苯基)-7a-甲基-5'-苯基-2',3a, 4',7a-四氢- $2 H$ 螺[苯并呋喃-3,3'-吡唑]-2,5(4H)-二酩(3ak): 白色固体, $33.3 \mathrm{mg}$, 产率 $85 \%$. m.p. $222 \sim 224{ }^{\circ} \mathrm{C}$; ${ }^{1} \mathrm{H}$ NMR $(300$ $\mathrm{MHz}, \mathrm{CDCl}_{3}$ ) $\delta: 7.64$ (ddd, $\left.J=9.8,6.9,4.6 \mathrm{~Hz}, 3 \mathrm{H}\right), 7.38$ 
$(\mathrm{dd}, J=5.1,1.8 \mathrm{~Hz}, 3 \mathrm{H}), 7.22 \sim 7.06(\mathrm{~m}, 3 \mathrm{H}), 6.83(\mathrm{dd}$, $J=10.4,1.6 \mathrm{~Hz}, 1 \mathrm{H}), 6.19$ (d, $J=10.4 \mathrm{~Hz}, 1 \mathrm{H}), 6.15 \sim$ $6.14(\mathrm{~m}, 1 \mathrm{H}), 3.41(\mathrm{dd}, J=38.7,17.3 \mathrm{~Hz}, 2 \mathrm{H}), 3.24(\mathrm{dd}$, $J=2.6 \mathrm{~Hz}, 1.8 \mathrm{~Hz}, 1 \mathrm{H}), 2.70$ (d, $J=4.4 \mathrm{~Hz}, 2 \mathrm{H}), 1.69$ (s, $3 \mathrm{H}) ; \quad{ }^{13} \mathrm{C} \mathrm{NMR}\left(151 \mathrm{MHz}, \mathrm{CDCl}_{3}\right) \delta: 207.0,194.6,173.9$, $153.2(\mathrm{~d}, J=242.1 \mathrm{~Hz}), 148.0,147.8,130.9,130.1$ (d, $J=$ $8.6 \mathrm{~Hz}), 129.7,129.3,128.8,126.1,125.4,125.23,125.17$, 124.9, 116.2 (d, $J=21.0 \mathrm{~Hz}), 78.5,74.7,44.1,41.6,33.4$, 31.0, 26.4; HRMS (ESI) calcd for $\mathrm{C}_{23} \mathrm{H}_{20} \mathrm{FN}_{2} \mathrm{O}_{3}[\mathrm{M}+\mathrm{H}]^{+}$ 391.1452, found 391.1453.

2'-(2-氯苯基)-7a-甲基-5'-苯基-2',3a,4',7a-四氢- $2 H$ 螺 [苯并呋喃-3,3'-吡唑]-2,5(4H)-二酮(3al): 白色固体, $32.5 \mathrm{mg}$, 产率 80\%, m.p. $138 \sim 140{ }^{\circ} \mathrm{C} ;{ }^{1} \mathrm{H}$ NMR (300 $\left.\mathrm{MHz}, \mathrm{CDCl}_{3}\right) \delta: 7.65 \sim 7.52(\mathrm{~m}, 3 \mathrm{H}), 7.45(\mathrm{dd}, J=7.8,1.7$ $\mathrm{Hz}, 1 \mathrm{H}), 7.38 \sim 7.27(\mathrm{~m}, 4 \mathrm{H}), 7.23(\mathrm{dd}, J=7.5,1.7 \mathrm{~Hz}$, $1 \mathrm{H}), 6.78(\mathrm{dd}, J=10.4,1.7 \mathrm{~Hz}, 1 \mathrm{H}), 6.16(\mathrm{~d}, J=10.4 \mathrm{~Hz}$, $1 \mathrm{H}), 3.29$ (q, $J=16.8 \mathrm{~Hz}, 2 \mathrm{H}), 3.03$ (dd, $J=55.1,13.0 \mathrm{~Hz}$, 2H), $2.70(\mathrm{dd}, J=19.0,7.1 \mathrm{~Hz}, 1 \mathrm{H}), 1.60(\mathrm{~s}, 3 \mathrm{H}) ;{ }^{13} \mathrm{C}$ NMR $\left(75 \mathrm{MHz}, \mathrm{CDCl}_{3}\right) \delta: 194.4,173.0,148.3,147.4$, $139.5,131.4,130.9,129.94,129.84,129.1,129.0,128.3$, 128.1, 127.7, 125.6, 78.3, 75.7, 43.6, 40.4, 33.5, 30.6, 25.9; HRMS (ESI) calcd for $\mathrm{C}_{23} \mathrm{H}_{19} \mathrm{ClN}_{2} \mathrm{O}_{3} \mathrm{Na}[\mathrm{M}+\mathrm{Na}]^{+}$ 429.0976, found 429.0973.

2'-(3-氯苯基)-7a-甲基-5'-苯基-2',3a,4',7a-四氢- $2 H$ 螺[苯并呋喃-3,3'-吡唑]-2,5(4H)-二酮(3am): 白色固体, $36.7 \mathrm{mg}$, 产率 $90 \%$. m.p. $200 \sim 202{ }^{\circ} \mathrm{C} ;{ }^{1} \mathrm{H}$ NMR $(300$ $\left.\mathrm{MHz}, \mathrm{CDCl}_{3}\right) \delta: 7.60(\mathrm{dd}, J=6.5,3.2 \mathrm{~Hz}, 2 \mathrm{H}), 7.39$ (dd, $J=5.0,1.8 \mathrm{~Hz}, 4 \mathrm{H}), 7.22(\mathrm{~d}, J=8.1 \mathrm{~Hz}, 1 \mathrm{H}), 6.96 \sim 6.81$ $(\mathrm{m}, 3 \mathrm{H}), 6.20(\mathrm{~d}, J=10.4 \mathrm{~Hz}, 1 \mathrm{H}), 3.57 \sim 3.35(\mathrm{~m}, 3 \mathrm{H})$, $2.69 \sim 2.56(\mathrm{~m}, 2 \mathrm{H}), 1.79(\mathrm{~s}, 3 \mathrm{H}) ;{ }^{13} \mathrm{C} \mathrm{NMR}(75 \mathrm{MHz}$, $\left.\mathrm{CDCl}_{3}\right) \delta: 193.9,173.6,147.2,146.5,142.4,135.1,130.2$, $130.0,129.4,129.1,128.5,125.7,121.0,116.2,113.2$, 78.8, 72.9, 42.4, 41.1, 33.2, 25.7; HRMS (ESI) calcd for $\mathrm{C}_{23} \mathrm{H}_{20} \mathrm{ClN}_{2} \mathrm{O}_{3}[\mathrm{M}+\mathrm{H}]^{+}$407.1157, found 407.1151.

$2^{\prime}$-(3-溴苯基)-7a-甲基-5'-苯基-2',3a,4',7a-四氢- $2 H$ 螺[苯并呋喃-3,3'-吡唑]-2,5(4H)-二酮 (3an): 白色固体, $42.2 \mathrm{mg}$, 产率 94\%. m.p. $216 \sim 218{ }^{\circ} \mathrm{C}$; ${ }^{1} \mathrm{H}$ NMR (300 $\left.\mathrm{MHz}, \mathrm{CDCl}_{3}\right) \delta: 7.63 \sim 7.56(\mathrm{~m}, 2 \mathrm{H}), 7.42 \sim 7.36(\mathrm{~m}, 4 \mathrm{H})$, $7.20 \sim 7.06(\mathrm{~m}, 2 \mathrm{H}), 6.95(\mathrm{ddd}, J=8.1,2.3,1.1 \mathrm{~Hz}, 1 \mathrm{H})$, $6.84(\mathrm{dd}, J=10.4,1.7 \mathrm{~Hz}, 1 \mathrm{H}), 6.20$ (d, $J=10.9 \mathrm{~Hz}, 1 \mathrm{H})$, $3.56 \sim 3.34(\mathrm{~m}, 3 \mathrm{H}), 2.66(\mathrm{dd}, J=7.2,4.2 \mathrm{~Hz}, 2 \mathrm{H}), 1.79$ (s, $3 \mathrm{H}) ;{ }^{13} \mathrm{C} \mathrm{NMR}\left(75 \mathrm{MHz}, \mathrm{CDCl}_{3}\right) \delta: 193.9,173.5,147.2$, $146.5,142.5,130.2,129.5,129.1,128.5,125.7,123.9$, 123.2, 119.0, 113.6, 78.8, 72.8, 42.3, 41.1, 33.2, 25.6; HRMS (ESI) calcd for $\mathrm{C}_{23} \mathrm{H}_{20} \mathrm{BrN}_{2} \mathrm{O}_{3}[\mathrm{M}+\mathrm{H}]^{+} 453.0634$, found $451.0584,453.0636$.

$7 \mathrm{a}$-甲基-5'-苯基-2'-(邻甲基苯基)-2',3a,4',7a-四氢$2 H$-螺[苯并呋喃-3,3'-吡唑]-2,5(4H)-二酮(3ao): 白色固 体, $32.8 \mathrm{mg}$, 产率 85\%. m.p. 184 $186{ }^{\circ} \mathrm{C} ;{ }^{1} \mathrm{H}$ NMR $(300$ $\left.\mathrm{MHz}, \mathrm{CDCl}_{3}\right) \delta: 7.61 \sim 7.52(\mathrm{~m}, 2 \mathrm{H}), 7.34(\mathrm{dd}, J=4.8,2.5$ $\mathrm{Hz}, 3 \mathrm{H}), 7.23 \sim 7.13(\mathrm{~m}, 3 \mathrm{H}), 6.79(\mathrm{dd}, J=10.4,1.7 \mathrm{~Hz}$, $1 \mathrm{H}), 6.17$ (d, $J=10.3 \mathrm{~Hz}, 1 \mathrm{H}), 3.28$ (q, $J=16.9 \mathrm{~Hz}, 2 \mathrm{H})$, $3.04 \sim 2.98(\mathrm{~m}, 1 \mathrm{H}), 2.83 \sim 2.55(\mathrm{~m}, 2 \mathrm{H}), 2.36(\mathrm{~s}, 3 \mathrm{H})$, $1.58(\mathrm{~s}, 3 \mathrm{H}) ;{ }^{13} \mathrm{C} \mathrm{NMR}\left(75 \mathrm{MHz}, \mathrm{CDCl}_{3}\right) \delta: 194.2,173.8$, $147.5,145.6,140.2,135.2,131.5,131.2,128.9,128.7$, $128.2,126.5,126.3,125.3,125.1,78.3,74.9, \quad 43.3,40.1$, $33.5,25.8,19.2$; HRMS (ESI) calcd for $\mathrm{C}_{24} \mathrm{H}_{23} \mathrm{~N}_{2} \mathrm{O}_{3}[\mathrm{M}+$ $\mathrm{H}]^{+}$387.1703, found 387.1705 .

$7 \mathrm{a}$-甲基-5'-苯基-2'-(对甲基苯基)-2',3a,4',7a-四氢$2 H$-螺[苯并呋喃-3,3'-吡唑]-2,5(4H)-二酮(3ap): 白色固 体, $34.4 \mathrm{mg}$, 产率 89\%. m.p. 192 194 ${ }^{\circ} \mathrm{C} ;{ }^{1} \mathrm{H}$ NMR $(300$ $\left.\mathrm{MHz}, \mathrm{CDCl}_{3}\right) \delta: 7.63 \sim 7.53(\mathrm{~m}, 2 \mathrm{H}), 7.39 \sim 7.32(\mathrm{~m}, 3 \mathrm{H})$, 7.09 (t, $J=11.7 \mathrm{~Hz}, 4 \mathrm{H}), 6.81$ (dd, $J=10.4,1.6 \mathrm{~Hz}, 1 \mathrm{H})$, $6.17(\mathrm{~d}, J=10.4 \mathrm{~Hz}, 1 \mathrm{H}), 3.61 \sim 3.17(\mathrm{~m}, 3 \mathrm{H}), 2.63(\mathrm{~d}, J=$ $4.6 \mathrm{~Hz}, 2 \mathrm{H}), 2.32(\mathrm{~s}, 3 \mathrm{H}), 1.70(\mathrm{~s}, 3 \mathrm{H}) ;{ }^{13} \mathrm{C} \mathrm{NMR}(75 \mathrm{MHz}$, $\left.\mathrm{CDCl}_{3}\right) \delta: 194.2,174.1,147.3,145.5,139.3,131.5,130.8$, $129.7,129.0,128.9,128.4,125.5,117.3,78.5,73.7,42.4$, 40.8, 33.2, 25.6, 20.3; HRMS (ESI) calcd for $\mathrm{C}_{24} \mathrm{H}_{23} \mathrm{~N}_{2} \mathrm{O}_{3}$ $[\mathrm{M}+\mathrm{H}]^{+}$387.1703, found 387.1704.

$7 \mathrm{a}$-乙基-2',5'-二苯基-2',3a,4',7a-四氢-2H-螺[苯并呋 喃-3,3'-吡唑]-2,5(4H)-二酮(3ba): 白色固体, $30.0 \mathrm{mg}$, 产 率 78\%. m.p. 134 136 ${ }^{\circ} \mathrm{C} ;{ }^{1} \mathrm{H}$ NMR $\left(300 \mathrm{MHz}, \mathrm{CDCl}_{3}\right.$ ) $\delta: 7.89 \sim 7.81(\mathrm{~m}, 1 \mathrm{H}), 7.67 \sim 7.54(\mathrm{~m}, 2 \mathrm{H}), 7.46 \sim 7.29$ $(\mathrm{m}, 5 \mathrm{H}), 7.22 \sim 7.13(\mathrm{~m}, 2 \mathrm{H}), 7.06 \sim 6.97(\mathrm{~m}, 1 \mathrm{H}), 6.89 \sim$ $6.81(\mathrm{~m}, 1 \mathrm{H}), 6.26(\mathrm{~d}, J=10.5 \mathrm{~Hz}, 1 \mathrm{H}), 3.62 \sim 3.30(\mathrm{~m}$, $3 \mathrm{H}), 2.18 \sim 1.85(\mathrm{~m}, 2 \mathrm{H}), 1.08(\mathrm{t}, J=7.5 \mathrm{~Hz}, 3 \mathrm{H}) ;{ }^{13} \mathrm{C}$ NMR (75 MHz, $\left.\mathrm{CDCl}_{3}\right) \delta: 194.4,174.2,146.7,145.7$, $141.5,130.62,130.56,129.9,129.1,128.4,125.5,121.7$, $116.9,115.0,81.0,73.5,41.2,40.2$, 33.6, 32.2, 7.5; HRMS (ESI) calcd for $\mathrm{C}_{24} \mathrm{H}_{22} \mathrm{~N}_{2} \mathrm{O}_{3} \mathrm{Na}[\mathrm{M}+\mathrm{Na}]^{+} 409.1523$, found 409.1520 .

$2^{\prime}, 5^{\prime}, 7 \mathrm{a}$-三苯基-2',3a,4',7a-四氢- $2 H$-螺 [苯并呋喃3,3'-吡唑]-2,5(4H)-二酮(3ca): 淡黄色固体, $37.1 \mathrm{mg}$, 产 率 85\%. m.p. $168 \sim 170{ }^{\circ} \mathrm{C} ;{ }^{1} \mathrm{H}$ NMR $\left(300 \mathrm{MHz}, \mathrm{CDCl}_{3}\right)$ $\delta: 7.72 \sim 7.56(\mathrm{~m}, 2 \mathrm{H}), 7.54 \sim 7.35(\mathrm{~m}, 8 \mathrm{H}), 7.34 \sim 7.27$ $(\mathrm{m}, 2 \mathrm{H}), 7.25 \sim 7.15(\mathrm{~m}, 2 \mathrm{H}), 7.02 \sim 6.94(\mathrm{~m}, 1 \mathrm{H}), 6.91$ $(\mathrm{dd}, J=10.4,1.7 \mathrm{~Hz}, 1 \mathrm{H}), 6.54 \sim 6.39(\mathrm{~m}, 1 \mathrm{H}), 3.71 \sim 3.44$ $(\mathrm{m}, 3 \mathrm{H}), 2.77 \sim 2.55(\mathrm{~m}, 2 \mathrm{H}) ;{ }^{13} \mathrm{C} \mathrm{NMR}\left(75 \mathrm{MHz}, \mathrm{CDCl}_{3}\right)$ $\delta: 194.5,174.1,145.8,145.3,141.4,137.7,130.6,130.3$, $129.2,129.1,128.4,125.6,124.0,121.9,117.1,81.5,73.7$, 
44.4, 41.2, 32.8; HRMS (ESI) calcd for $\mathrm{C}_{28} \mathrm{H}_{22} \mathrm{~N}_{2} \mathrm{O}_{3} \mathrm{Na}$ $[\mathrm{M}+\mathrm{Na}]^{+}$457.1523, found 457.1540.

\section{辅助材料(Supporting Information) 化合物 3aa $\sim 3 \mathbf{a q}$ 的 ${ }^{1} \mathrm{H}$ NMR 和 ${ }^{13} \mathrm{C} \mathrm{NMR}$ 谱图以及化合物 $3 \mathbf{a a}$ 的 $\mathrm{X}$ 射线 单晶衍射结构. 这些材料可以免费从本刊网站(http:// sioc-journal.cn/)上下载.}

\section{References}

[1] (a) Yoshida, H.; Yanai, H.; Namiki, Y.; Fukatsu-Sasaki, K.; Furutani, N.; Tada, N. CNS Drug Rev. 2006, 12, 9.

(b) Bhutani, R.; Pathak, D. P.; Husain, A.; Kapoor, G.; Kant, R. Int. J. Pharm. Sci. Res. 2015, 6, 4113.

(c) Marella, A.; Ali, M. R.; Alam, M. T.; Saha, R.; Tanwar, O.; Akhter, M.; Shaquiquzzaman, M.; Alam, M. M. Mini-Rev. Med. Chem. 2013, 13, 921.

(d) Li, Z.; Zhang, Z.; Zhang, W.; Liu, Q.; Liu, T.; Zhang, G. Synlett 2013, 24, 2735.

(e) Hong, Y.; Dai, H.; Ye, L.; Zhong, S.; Cao, X.; Shi, Y.; Li, C.; Shi, J.; Shi, L. Chin. J. Org. Chem. 2017, 37, 3006 (in Chinese). (洪宇, 戴红, 叶林玉, 仲苏林, 曹雄飞, 石玉军, 李春建, 石健, 施䂞, 有机化学, 2017, 37, 3006.)

(f) Sun, N.; Shen, Z.; Zhai, Z.; Han, L.; Weng, J.; Tan, C.; Liu, X. Chin. J. Org. Chem. 2017, 37, 2705 (in Chinese).

(孙娜波, 沈钟华, 翟志文, 韩亮, 翁建全, 谭成侠, 刘幸海, 有 机化学, 2017, 37, 2705.)

(g) Dai, H.; Yao, W.; Ye, L.; Fang, Y.; Shi, Y.; Song, C.; Li, C.; Shi, J. Chin. J. Org. Chem. 2017, 37, 2165 (in Chinese).

(戴红, 姚炜, 叶林玉, 方源, 石玉军, 宋婵, 李春建, 石健, 有 机化学, 2017, 37, 2165.)

(h) Shi, J.; Ren, G.; Wu, N.; Liu, X.; Xu, T.; Tan, C. Chin. J. Org. Chem. 2017, 37, 2131 (in Chinese).

(史建俊, 任贵华, 吴宁捷, 刘幸海, 许天明, 谭成侠, 有机化学, 2017, 37, 2131.)

(i) Sun, N.; Shen, Z.; Zhai, Z.; Wu, H.; Weng, J.; Tan, C.; Liu, X. Chin. J. Org. Chem. 2017, 37, 2044 (in Chinese).

(孙娜波, 沈钟华, 翟志文, 武宏科, 翁建全, 谭成侠, 刘幸海, 有机化学, 2017, 37, 2044.)

(j) Shi, Y.; Ye, L.; Zhong, S.; Cao, X.; Dai, H.; Hong, Y.; Li, C.; Shi, J.; Wu, J. Chin. J. Org. Chem. 2017, 37, 1844 (in Chinese).

(石玉军, 叶林玉, 仲苏林, 曹雄飞, 戴红, 洪宇, 李春建, 石健, 吴锦明, 有机化学, 2017, 37, 1844.)

(k) Dai, H.; Chen, J.; Hong, Y.; Yuan, B.; Fan, C.; Ma, R.; Liang, Z.; Shi, J. Chin. J. Org. Chem. 2017, 37, 1542 (in Chinese). (戴红, 陈佳, 洪宇, 袁斌颖, 范崇光, 马瑞媛, 梁志鹏, 石健, 有机化学, 2017, 37, 1542.)

[2] Ivachtchenko, A. V.; Ivanenkov, Y. A.; Mitkin, O. D.; Vorobiev, A. A.; Kuznetsova, I. V.; Shevkun, N. A.; Koryakova, A. G.; Karapetian, R. N.; Trifelenkov, A. S.; Kravchenko, D. V.; Veselov, M. S.; Chufarova, N. V. Eur. J. Med. Chem. 2015, 99, 51.

[3] Merino-Montiel, P.; López, O.; Álvarez, E.; Fernández-Bolañosa, J. G. Tetrahedron 2012, 68, 4888 .

[4] Gasch, C.; Merino-Montiel, P.; López, O.; Fernández-Bolañosa, J. G.; Fuentes, J. Tetrahedron 2010, 66, 9964.

[5] Kamal, A.; Reddy, B. V. S.; Sridevi, B.; Ravikumar, A.; Venkateswarlu, A.; Sravanthi, G.; Sridevi, J. P.; Yogeeswari, P.; Sriram, D. Bioorg. Med. Chem. Lett. 2015, 25, 3867.

[6] Huisgen, R.; Fleischmann, R.; Eckell, A. Tetrahedron Lett. 1960, 12,5 .

[7] Belletire, J. L.; Hagedom, C. E.; Ho, D. M.; Krause, J. Tetrahedron Lett. 1993, 34, 797.

[8] Mei, L. Y.; Tang, X. Y.; Shi, M. Chem.-Eur. J. 2014, 20, 1.

[9] Hashimoto, T.; Maruoka, K. Chem. Rev. 2015, 115, 5366.

[10] (a) Gothelf, K. V.; Jørgensen, K. A. Chem. Rev. 1998, 98, 863. (b) Lima, C. G. S.; Ali, A.; Berkel, S. S. V.; Westermann, B.; Paixão, M. W. Chem. Commun. 2015, 51, 10784.

(c) Narayan, R.; Potowski, M.; Jia, Z.; Antonchick, A. P.; Waldmann, H. Acc. Chem. Res. 2014, 47, 1296.

(d) Fustero, S.; Rosell, M. S.; Barrio, P.; Fuentes, A. S. Chem. Rev. 2011, 111, 6984.

(e) Fanga, X.; Wang, C. J. Org. Biomol. Chem. 2018, 16, 2591.

[11] (a) Callaghan, P. D.; Elliott, A. J.; Gibson, M. S. J. Org. Chem. 1975, 40, 2131.

(b) Padwa, A.; Nahm, S.; Sato, E. J. Org. Chem. 1978, 43, 1664

(c) Sasaki, T.; Eguchi, S.; Toi, N. J. Org. Chem. 1979, 44, 3711.

(d) Shine, H. J.; Hoque, A. K. M. M. J. Org, Chem. 1988, 53, 4349.

(e) Shawali, A. S.; Mosselhi, M. A. N.; Tawfik, N. M. J. Org. Chem. 2001, 66, 4055.

(f) Sibi, M. P.; Stanley, L. M.; Soeta, T. Adv. Synth. Catal. 2006, $348,2371$.

(g) Spiteri, C.; Keeling, S.; Moses, J. E. Org. Lett. 2010, 12, 3368.

(h) Riyadh, S. M.; Farghaly, T. A. Tetrahedron 2012, 68, 9056.

(i) Milen, M.; Balogh, P. A.; Dancsó, A.; Keglevich, G. Synthesis 2012, 44, 3447.

(j) Sayed, A. R. Tetrahedron 2013, 69, 5293.

(k) Liu, B.; Li, X. F.; Liu, H. C.; Yu, X. Y. Tetrahedron Lett. 2013 , 54, 6952 .

(1) Gerten, A. L.; Slade, M. C.; Pugha, K. M.; Stanley, L. M. Org. Biomol. Chem. 2013, 11, 7834.

(m) Dadiboyena, S.; Valente, E. J.; Hamme II, A. T. Tetrahedron Lett. 2014, 55, 2208.

(n) Dadiboyena S.; Hamme II, A. T. Eur. J. Org. Chem. 2013, 7567. (o) Hu, X. L.; Liu, B.; Liu, H. C.; Li, X. F. Chin. J. Org. Chem. 2013, 33, 1583 (in Chinese).

(胡小莲, 刘涁, 刘浩冲, 李符芳, 有机化学, 2013, 33, 1583.)

(p) Alizadeh, A.; Moafi, L.; Ghanbaripour, R.; Abadi, M. H.; Zhu, Z.; Kubicki, M. Tetrahedron 2015, 71, 3495.

(q) Garve, L. K. B.; Petzold, M.; Jones, P. G.; Werz, D. B. Org. Lett. 2016, $18,564$.

(r) Blackburn, J.; Molyneux, G.; Pitard, A.; Rice, C. R.; Page, I. M.; Afshinjavid, S.; Javid, F. A.; Coles, S. J.; Horton, P. N.; Hemming, K. Org. Biomol. Chem. 2016, 14, 2134.

(s) Soeta, T.; Takashita, S.; Sakata, Y.; Ukaji, Y. Asian J. Org. Chem. 2016, 5, 1041.

(t) Alizadeh, A.; Moafi, L. Synlett 2016, 27, 1828.

(u) Liew, S. K.; Holownia, A.; Tilley, A. J.; Carrera, E. I.; Seferos, D. S.; Yudin, A. K. J. Org. Chem. 2016, 81, 10444.

(v) Guo, C.-X.; Zhang, W.-Z.; Zhang, N.; Lu, X.-B. J. Org. Chem. 2017, 82, 7637 .

(w) Ribeiro, C. J. A.; Nunes, R. C.; Amaral, J. D.; Gonçalves, L. M.; Rodrigues, C. M. P.; Moreira, R.; Santos, M. M. M. Eur. J. Med. Chem. 2017, 140, 494.

(x) Alizadeh, A.; Moafi, L. Heterocycl. Commun. 2017, 23, 375.

(y) Zhao, H.-W.; Zhao, Y.-D.; Liu, Y.-Y.; Zhao, L.-J.; Song, X.-Q.; Chen, X.-Q.; Pang, H.-Q.; Du, J.; Feng, N.-N. RSC Adv. 2017, 7, 55106.

(z) Gardias, A.; Kaszyński, P.; Obijalska, E.; Trzybiński, D.; Domagała, S.; Woźniak, K.; Szczytko, J. Chem.-Eur. J. 2018, 24, 1317.

[12] Huisgen, R.; Grashey, R.; Seidel, M.; Wallbilllich, G.; Knupfer, H.; Schmidt R. Justus Liebigs Ann. Chem. 1962, 653, 105.

[13] Tsuge, O.; Watanabe, H.; Kiryu, Y. Bull. Chem. Soc. Jpn. 1979, 52, 3654.

[14] Gandolfi, R.; Toma, L. Tetrahedron 1979, 36, 935.

[15] (a) Guo, Z.; Jia, H.; Liu, H.; Wang, Q.; Huang, J.; Guo, H. Org. Lett. 2018, 20, 2939.

(b) Long, W.; Chen, S.; Zhang, X.; Fang, L.; Wang, Z. Tetrahedron 2018, 74, 6155 .

[16] Wang, B.; Wang, Q. J.; Liu, H. L.; Liao, J. N.; Huang, J. X.; Guo, H. Chin. J. Org. Chem. 2019, 39, 1354 (in Chinese). (王博, 王奇君, 刘洪蕾, 廖健宁, 黄家兴, 郭红超, 有机化学, 2019, 39, 1354.) 
[17] (a) Na, R.; Jing, C.; Xu, Q.; Jiang, H.; Wu, X.; Shi, Y.; Zhong, J.; Wang, M.; Benitez, D.; Tkatchouk, E.; Goddard III, W. A.; Guo, H.; Kwon, O. J. Am. Chem. Soc. 2011, 133, 13337.

(b) Wu, X.; Na, R.; Liu, H.; Liu, J.; Wang, M.; Zhong, J.; Guo, H. Tetrahedron Lett. 2012, 53, 342.

(c) Guo, H.; Liu, H.; Zhu, F.-L.; Na, R.; Jiang, H.; Wu, Y.; Zhang, L.; Li, Z.; Yu, H.; Wang, B.; Xiao, Y.; Hu, X.-P.; Wang, M. Angew. Chem., Int. Ed. 2013, 52, 12641

(d) Zhang, L.; Yu, H.; Yang, Z.; Liu, H.; Li, Z.; Guo, J.; Xiao, Y.; Guo, H. Org. Biomol. Chem. 2013, 11, 8235.

(e) Liu, H.; Wu, Y.; Zhao, Y.; Li, Z.; Zhang, L.; Yang, W.; Jiang, H.; Jing, C.; Yu, H.; Wang, B.; Xiao, Y.; Guo, H. J. Am. Chem. Soc. 2014, 136, 2625.

(f) Zhang, L.; Liu, H.; Qiao, G.; Hou, Z.; Liu, Y.; Xiao, Y.; Guo, H. J. Am. Chem. Soc. 2015, 137, 4316.

(g) Liu, H.; Yuan, C.; Wu, Y.; Xiao, Y.; Guo, H. Org. Lett. 2015, 17, 4220 .

(h) Gao, Z.; Wang, C.; Yuan, C.; Zhou, L.; Xiao, Y.; Guo, H. Chem. Commun. 2015, 51, 12653.

(i) Yang, W.; Zhang, Y.; Qiu, S.; Zhao, C.; Zhang, L.; Liu, H.; Zhou, L.; Xiao, Y.; Guo, H. RSC Adv. 2015, 5, 62343.

(j) Liu, Y.; Yang, W.; Wu, Y.; Mao, B.; Gao, X.; Liu, H.; Sun, Z.; Xiao, Y.; Guo, H. Adv. Synth. Catal. 2016, 358, 2867.

(k) Wang, C.; Gao, Z.; Zhou, L.; Yuan, C.; Sun, Z.; Xiao, Y.; Guo, H. Org. Lett. 2016, 18, 3418.

(1) Yuan, C.; Zhou, L.; Xia, M.; Sun, Z.; Wang, D.; Guo, H. Org.
Lett. 2016, 18, 5644.

(m) Li, Z.; Yu, H.; Liu, Y.; Zhou, L.; Sun, Z.; Guo, H. Adv. Synth. Catal. 2016, 358, 1880.

(n) Wang, C.; Jia, H.; Zhang, C.; Gao, Z.; Zhou, L.; Yuan, C.; Xiao, Y.; Guo, H. J. Org. Chem. 2017, 82, 633.

(o) Yang, W.; Sun, W.; Zhang, C.; Wang, Q.; Guo, Z.; Mao, B.; Liao, J.; Guo, H. ACS Catal. 2017, 7, 3142.

(p) Zhou, L.; Yuan, C.; Zhang, C.; Zhang, L.; Gao, Z.; Wang, C.; Liu, H.; Wu, Y.; Guo, H. Adv. Synth. Catal. 2017, 359, 2316.

(q) Wang, B.; Liu, H.; Wang, Q.; Yuan, C.; Jia, H.; Liu, C.; Guo, H. Tetrahedron 2017, 73, 5926.

(r) Liu, H.; Zhao, Y.; Li, Z.; Jia, H.; Zhang, C.; Xiao, Y.; Guo, H. RSC Adv. 2017, 7, 29515.

(s) Gao, Z.; Wang, C.; Zhou, L.; Yuan, C.; Xiao, Y.; Guo, H. Org. Lett. 2018, 20, 4302.

(t) Zhou, L.; Yuan, C.; Zeng, Y.; Liu, H.; Wang, C.; Gao, X.; Wang, Q.; Zhang, C.; Guo, H. Chem. Sci. 2018, 9, 1831.

(u) Yuan, C.; Wu, Y.; Wang, D.; Zhang, Z.; Wang, C.; Zhou, L.; Zhang, C.; Song, B.; Guo, H. Adv. Synth. Catal. 2018, 360, 652.

(v) Jia, H.; Guo, Z.; Liu, H.; Mao, B.; Shi, X.; Guo, H. Chem. Commun. 2018, 54, 7050 .

[18] Takizawa, S.; Nguyen, T. M. N.; Grossmann, A.; Enders, D.; Sasai, H. Angew. Chem., Int. Ed. 2012, 51, 5423.

[19] Liu, H. L.; Jia, H.; Wang, B; Xiao, Y.; Guo; H. Org. Lett. 2017, 19, 4714 . 